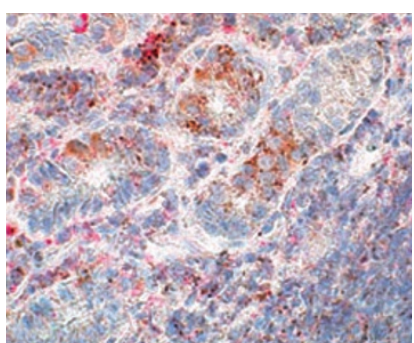

p 495

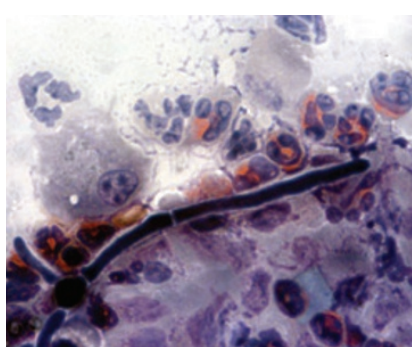

p 504

\section{ICMI 2009}

Charlotte Kaetzel highlights some of the recent discoveries discussed at the 14th International Congress of Mucosal Immunology, with a focus on commensal microbiota and the mucosal immune system. See page 464

\section{Waste management}

Peter Ernst comments on the book The Big Necessity: The Unmentionable World of Human Waste and Why It Matters. See page 466

\section{Update on IgA B-cell differentiation}

Keiichiro Suzuki and Sidonia Fagarasan provide an insightful commentary on the ongoing debate regarding where and how immunoglobulin Aproducing $B$ cells are generated, including the role of lymphoid tissue inducer cells in this process.

See page 468

\section{LTi cells in the evolution of adaptive immunity}

Peter Lane and colleagues discuss of the origin and function of lymphoid tissue inducer cells and the roles these cells play in driving immune responses and in the formation of lymphoid structures. See page 472

\section{Lymphoid tissues at the interface}

Gérard Eberl and Matthias Lochner review the factors influencing the development of the diverse lymphoid tissues in the intestine and how they have evolved to regulate immunity to self-antigens, commensal bacteria, and bacterial pathogens.

See page 478

\section{Inflammatory attraction for LTi cells}

Federica Marchesi and colleagues demonstrate that overexpression of CXCL13 in intestinal epithelial cells, as occurs with intestinal inflammation, results in accumulation of Ti cells, B cells, and natural killer cells, as well as in an increase in the number and size of lymphoid follicles in intestinal tissue.

See page 486

\section{IgA class switching in humans}

Francesca Barone and colleagues find evidence for T-cell-dependent and -independent immunoglobulin A class-switch recombination in the organized gut-associated lymphoid tissues.

See page 495

\section{Fungal proteinases trigger asthma}

Paul Porter and colleagues report that proteinases released by common environmental fungi that can infect mammalian airway mucosa can trigger asthmalike disease. See page 504

\section{Circulating $\alpha 4 \beta 7^{+} \mathrm{CD} 4^{+} \mathrm{T}$ cells in SIV}

Xiaolei Wang and colleagues show a good correlation between the loss of $\alpha 4 \beta 7^{+} \mathrm{CD} 4^{+}$cells in the blood and the loss of this cell type in the intestine in macaques with SIV infection. These observations may prove useful for monitoring disease pathogenesis in HIV patients. See page 518

\section{IELs contribute to T. gondii ileitis}

Charlotte Egan and colleagues reveal an active contribution by intraepithelial lymphocytes to the gut pathology induced by intestinal Toxoplasma gondii infection. See page 527

\section{Nasal vaccination for SIV}

Using a DNA-MVA (modified vaccinia virus Ankara) boost approach for the prevention of SIV in macaques, Mariana Manrique and colleagues demonstrate that nasal vaccination induces stronger and more lasting protection against AIDS than does intramuscular vaccination. See page 536 